\title{
Chronotropic incompetence: a proposal for definition and diagnosis
}

\author{
Department of \\ Cardiological \\ Sciences, St George's \\ Hospital Medical \\ School, London \\ D Katritsis \\ A J Camm \\ Correspondence to: \\ Dr D Katritsis, Department \\ of Cardiological Sciences, St \\ Georges's Hospital Medica \\ School, Cranmer Terrace, \\ London SW17 ORE. \\ Accepted for publication \\ 26 April 1993
}

\author{
Demosthenes Katritsis, A John Camm
}

Between 1958 and 1960 Astrand documented the normal heart rate response to exercise in healthy individuals and noted that the maximum heart rate decreased with age. ${ }^{12}$ A reduced cardiac chronotropic response to isoprenaline has been reported in elderly subjects and alterations in catecholamineadrenergic receptor interactions may be responsible for this change of cardiovascular regulation in the elderly. ${ }^{3-5}$ In addition the parasympathetic innervation of the sinus node is currently under investigation. ${ }^{6}$ Over the years it has also been recognised that an inadequate chronotropic response (chronotropic incompetence) at maximal exercise is common in patients with cardiac disease $^{78}$ and much interest has centred on the pathophysiological mechanism(s) involved in chronotropic incompetence..$^{10}$ Despite this, no universally accepted definition of chronotropic incompetence exists and the criteria for its diagnosis are not defined. However, a precise diagnosis of this condition is of clinical importance. First, adaptive-rate pacing may be beneficial in patients who clearly demonstrate chronotropic incompetence. ${ }^{11-14}$ Secondly, chronotropic incompetence has also been documented in patients with chronic heart failure and may contribute to the impairment of physical capacity seen in these patients. ${ }^{715}$

\section{Terminology and definitions}

The term chronotropic incompetence implies the inability of the heart to increase its rate in proportion to metabolic demand. Consequently, it refers either to inadequacy of the sinus node or, in the case of complete heart block, of the lower escape pacemaker to respond to exercise or other autonomic changes. Unfortunately in published reports a clear distinction is not always made between chronotropic incompetence of the sinus node and of the lower escape pacemaker.

The current definitions of chronotropic incompetence usually rely on the heart rate response achieved at maximal exercise. The expression which was formulated by Astrand to predict the age dependent maximum sinus rate response to exercise (maximum predicted heart rate, MPHR) was $M P H R=$ (220-age) beats/min and chronotropic incompetence has been arbitrarily defined as a maximum exercise heart rate achieved during exercise testing that is $<75 \%{ }^{16}$ or $<80 \%{ }^{17}$ of the predicted MPHR. Other arbitrary definitions such as a maximum exercise heart rate $<100$ beats $/ \mathrm{min}^{11}$ or $<120$ beats $/ \mathrm{min}^{18}$ have also been used. However, the achievement of maximum exercise is not always possible. Elderly cardiac patients, especially those disabled by chronotropic incompetence, are unable to perform sufficient exercise on the treadmill. Furthermore, everyday life activities of these patients usually correspond to low work loads up to 6 metabolic equivalents corresponding to the first stage of the standard Bruce protocol. In addition, not much is known about the patterns of heart rate acceleration and deceleration in the presence of chronotropic incompetence.

Wilkoff et $a l^{19}$ using exercise testing with respiratory gas analysis and employing a less demanding protocol, the so-called Chronotropic Assessment Exercise Protocol (CAEP), recently established a method by which the chronotropic response during submaximal exercise can be assessed. This was achieved by comparing relative metabolic levels with relative heart rates-that is, by normalising the change in heart rate from rest to maximal exertion as a linear function of the change in metabolic workload. The normal predicted heart rate at some submaximal stage of exercise, regardless of the protocol used, is given by the formula:

$$
\begin{gathered}
\mathrm{HR}_{\text {stage }}=\left[\left(220 \text {-age-HR } \mathrm{HR}_{\text {rest }}\right) \times\right. \\
\left.\left(\mathrm{METS}_{\text {stage }}-1\right) /\left(\mathrm{METS}_{\text {peak }}-1\right)\right]+\mathrm{HR}_{\text {rest }}
\end{gathered}
$$

where $\operatorname{METS}=\mathrm{VO}_{2}(\mathrm{ml} / \mathrm{kg} / \mathrm{min}) / 3 \cdot 5, \quad$ and METS $_{\text {peak }}=$ the peak functional capacity achieved during the test.

This formula is proposed as a guide to the expected heart rate at various stages of exercise in normal subjects able to exercise to maximum workload, but it gives no idea as to the expectation in patients who are unable to achieve anaerobic peak exercise. In these patients the application of the formula will produce an exaggerated estimate of chronotropic incompetence during submaximal exercise because of its reliance on METS $_{\text {peak }}$ as a denominator in the equation. Wilkoff's methodology has been recently used by $\mathrm{Kay}^{20}$ in a proposed model for assessment of the pacing rates offered by adaptive-rate pacemakers from the beginning of exercise to complete recovery. 


\section{Prevalence}

The prevalence of this condition in most patient populations is not known and the inconsistency in defining chronotropic incompetence in the various reported studies makes comparative analysis of the existing data difficult. It has been suggested that approximately $40 \%$ of the pacemaker population exhibit some degree of chronotropic incompetence and might benefit from rate-adaptive pacing ${ }^{21}$ and that this percentage increases with time after implantation. ${ }^{17}$ The prevalence of chronotropic incompetence in sick sinus syndrome (due to inadequate response of the sinus node), defined as $<120$ beats $/ \mathrm{min}$ at maximum exercise, has been reported to range from $28 \%$ to $57 \% .{ }^{18}$ Characteristically, the definition of chronotropic incompetence in the published series is not always clear. ${ }^{22}$ In atrial fibrillation (usually due to inadequate response of the atrioventricular node conduction) the chronotropic response to exercise may be impaired in up to $60 \%$ of the patients. ${ }^{23}$ In patients with acquired complete heart block (usually due to inadequate response of the His-Purkinje pacemaker) chronotropic incompetence is almost universal. Chronotropic incompetence is not only important in patients with primary conduction disease but is also common in patients with heart failure whether this is caused by ischaemic heart disease ${ }^{15}$ or dilated or hypertrophic cardiomyopathy. ${ }^{24}$ Studies are currently underway to determine the true prevalence of chronotropic incompetence in such patients and to assess whether the restoration of chronotropic competence is of therapeutic benefit.

\section{Proposals}

DEFINITIONS

A standard definition of chronotropic incompetence is proposed based on an anatomical and a physiological differentiation. From an anatomical perspective chronotropic incompetence is divided into sinus nodal, junctional, or ventricular. Whereas from a physiological perspective we have proposed ${ }^{25}$ a distinction between two varieties: specific or functional.

Chronotropic incompetence is the inability of the heart rate to achieve at least $80 \%$ (an arbitrary percentage for the time being) of the predicted value according to Astrand's formula (220- age) at peak exercise-that is, achievement of the anaerobic threshold. Inability of the heart rate to achieve at least $80 \%$ of the predicted value according to Wilkoff's formula at any stage of submaximal exercise may also denote a form of chronotropic incompetence that at present is particularly relevant to the pacing rate modulation offered by adaptive-rate pacemakers. ${ }^{20}$

Sinus node chronotropic incompetence is the inability of the sinus node to achieve at least $80 \%$ of the predicted rate. Specific sinus node chronotropic incompetence is the inability of the sinus node to accelerate in response to metabolic demands secondary to intrinsic disease or negative chronotropic drugs. Functional chronotropic incompetence manifests itself either as atrial tachyarrhythmias or as retrograde ventriculoatrial conduction resulting in sinus node reset, pacemakermediated tachycardia, or the so-called atrioventricular desynchronisation arrhythmia (that is, unsensed retrograde $P$ waves followed by ineffectual atrial stimulation during atrial refractoriness ${ }^{26}$ ). In such cases there is interference with sinus node activity preventing an appropriate sinus node rate response to exercise or autonomic changes.

functional chronotropic incompetence in patients with permanent atrial fibrillation implies inability of the heart rate to achieve $80 \%$ of the predicted values owing to the atrioventricular conduction defect. Also in patients with complete heart block it refers to the inability of the escape junctional pacemaker to achieve at least $80 \%$ of the predicted value. In cases of an intra-hisian or infra-hisian block the term ventricular chronotropic incompetence is more appropriate-to denote incompetence of the escape focus.

\section{DIAGNOSIS}

Ideally, cardiopulmonary exercise testing with respiratory gas analysis is required for a proper assessment of the chronotropic response, though this facility is not universally available. We propose the following practical scheme:

(1) If patients achieve more than $80 \%$ of their predicted maximum heart rate during an ordinary exercise test chronotropic incompetence is not present.

(2) In patients unable to achieve at least $80 \%$ of their predicted maximum heart rate on exercise, exercise testing with respiratory gas analysis should be performed, particularly in patients with abnormal left ventricular function.

(a) Patients who achieve the anaerobic threshold are chronotropically incompetent.

(b) In those patients who do not reach the anaerobic threshold at peak exercise, submaximal chronotropic incompetence at any stage of submaximal exercise can be assessed according to Wilkoff's formula. Since the

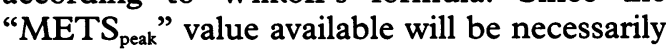
smaller than the one that corresponds to the anaerobic threshold, chronotropic incompetence can be excluded if the heart rate response is higher than $80 \%$ of that predicted according to the formula. If the achieved heart rate response is less than $80 \%$ of that predicted, chronotropic incompetence cannot be excluded.

1 Astrand I. The physical work capacity of workers 50-64 years old. Acta Physiol Scand 1958;42:73-86.

2 Astrand I. Aerobic work capacity in men and women with special reference to age. Acta Physiol Scand 1960;49: $1-92$.

3 Buhler FR, Bolli P. Changes in cardiovascular responsiveness caused by age and high blood pressure: Implications for therapy. $\mathcal{f}$ Cardiovasc Pharmacol 1985; 
7(suppl 3):206-15.

4 Vestal RE, Wood AJJ, Shand DG. Reduced $\beta$-adrenoreceptor sensitivity in the elderly. Clin Pharmacol Ther 1979;26:181-6.

5 Van Brummelen P, Buhler FR, Kiowski W, Amann FW. Age-related decrease in cardiac and peripheral vascular responsiveness to isoproterenol: Studies in normal subjects. Clin Sci 1981;60:571-7.

6 Carlson MD, Geha AS, Hsu J, Martin PJ, Levy MN, Jacobs G, Waldo AL. Selective stimulation of parasympathetic nerve fibers to the human sinoatrial node. Circulation 1992;85:1311-7.

7 Colucci WS, Ribeiro JP, Rocco MB, et al. Impaired chronotropic response to exercise in patients with congestive heart failure. Role of postsynaptic betaadrenergic desensitization. Circulation 1989;80:314-23.

8 Katritsis D, Jones S, Camm AJ: A rational choice of pacemaker mode. Eur $f$ Cardiac Pacing Electrophysiol maker mode.

9 Brown JE, McLeod AA, Shand DG. In support of cardiac chronotropic beta 2 adrenoceptors. Am $f$ Cardiol 1986;57:11f-16f

10 Hammond HK, Ransnas LA, Insel PA. Noncoordinate regulation of cardiac Gs protein and beta-adrenergic receptors by a physiological stimulus, chronic dynamic exercise. $f$ Clin Invest 1988;82:2168-71.

11 Batey R, Sweesy M, Scala G, Forney RC. Comparison of low rate dual chamber pacing to activity responsive rate variable ventricular pacing. $P A C E$ 1990;13:646-52.

12 Higano ST, Hayes DL, Eisinger G. Hemodynamic importance of AV synchrony during low levels of exercise tance of AV synchrony during
[abstr]. PACE 1989;12:1565.

13 Cazzin R, Capucci A, Zarbo F, Artusi L, Boriani G, Zanuttini D, Pascotto P, Piccolo E. Evaluation of rateresponsiveness benefit in patients with and without responsiveness benefit in patients with and without

14 Haywood G, Katritsis D, Ward J, Leigh-Jones M, Ward $\mathrm{DE}$, Camm AJ. Atrial adaptive rate pacing in sick sinus syndrome: effects on exercise capacity and atrial arrhythmias. Br Heart $\mathcal{F}$ 1993;69:174-8.
15 Yamabe $\mathrm{H}$, Kobayashi $\mathrm{K}$, Takata $\mathrm{T}$, Fukuzaki $\mathrm{H}$. Reduced chronotropic reserve to the metabolic requireReduced chronotropic reserve to the metabolic requirement during exercise in advanced heart failure with infarction. fpn Circ f 1987;51:259-64.

16 Isaeff DM, Jutzy RV, Florio J. Programming of rate responsive pacemakers. $f$ Electrophysiol 1989;3: 2102-216.

17 Gwinn N, Leman R, Zile $M$, et al. Pacemaker patients become chronotropic incompetent with time [abstr]. PACE 1990;13:535.

18 Rosenqvist $M$. Atrial pacing for sick sinus syndrome. Clin Cardiol 1990;13:43-47.

19 Wilkoff BL, Corey J, Blackburn G. A mathematical model of the exercise cardiac chronotropic response to exercise. 7 Electrophysiol 1989;3:176-80.

20 Kay GN. Quantitation of chronotropic response: comparison of methods for rate-modulating permanent paceson of methods for rate-modulating perm
makers. $7 \mathrm{Am}$ Coll Cardiol 1992;20:1533-41.

21 McBride JW, Reyes WJ, Medellin L. What is the need for rate modulated pacemakers? [abstr]. PACE 1990;13: 504.

22 Ryden L. Atrial inhibited pacing-An underused mode of cardiac stimulation. $P A C E$ 1988;11:1375-9

23 Corbelli R, Masterson M, Wilkoff BL. Chronotropic response to exercise in patients with atrial fibrillation. PACE 1990;13:179-85.

24 Keeling P, Haywood G, Klare E, Jennison S, Bent S, McKenna W. Chronotropic incompetence in chronic heart failure and its association with reduced exercise capacity in patients with dilated cardiomyopathy [abstr]. capacity in patients ath dilated cardiomyopathy [abstr]. Proceeding of the 13th Annual Scientific Session of the North American Society of
Electrophysiology. PACE 1992;15:575.

25 Camm AJ, Katritsis D. VVIR pacing is preferable to VDD pacing: when and why? In: Santini $M$, Pistolese $M$, pacing: when and why? In: Santini M, Pistolese M, Alliegro A, eds. Progress in clinica

26 Barold SS. Repetitive reentrant and non-reentrant ventriculoatrial synchrony in dual chamber pacing. Clin Cardiol 1991;14:754-63. 